resistance band mounted device offering objective measurement of adherence. It is valid and reliable for measuring exercise quality (time-under-tension (TUT)) and quantity (number of repetitions) (Rathleff et al., 2013a and b). The aim was to objectively evaluate adherence (repetitions and TUT) to an exercise intervention for RCRSP, and compare with self-report diaries.

Materials and Methods Ethical approval was obtained.

Adults ( $\geq 18$ years old) with RCRSP ( $\geq 6$ weeks) were eligible for inclusion. All participants provided written informed consent before being prescribed a 6 week home exercise programme. Participants were instructed to complete exercises daily, doing 10 repetitions per set with TUT of $8 \mathrm{~s}$ per repetition. Participants completed the NRS(pain) and SPADI questionnaire at baseline and 6 weeks.

Results 17 participants were eligible, with 11 participant data sets included in the analysis. There was on average 52\% adherence to the prescribed TUT. The adherence to repetitions was superior at $76 \%$, comparable to self-reported adherence (79\%). Half (6/11) of the participants reported changes in pain and disability scores which exceeded the minimal detectable change.

Conclusion The BandCizer ${ }^{\mathrm{TM}}$ was effective in quantifying adherence to TUT and repetitions, with poor adherence to TUT noted. Despite varied adherence, the intervention still produced significant changes in pain and disability. These findings support the need for further research examining parameters of exercise dosage in relation to patient outcomes in RCRSP.

\section{DIABETES MELLITUS AFFECT THE PROGNOSIS OF FROZEN SHOULDER: A 2 YEAR FOLLOW-UP STUDY}

${ }^{1}$ Per Gundtoft* ${ }^{2}$ Mikkel Lindegaard Attrup, ${ }^{3}$ Anne Krog Kristensen, ${ }^{3}$ Jette Wessel Vobbe, ${ }^{2}$ per Hölmich, ${ }^{3}$ Lilli Sørensen. ${ }^{1}$ Ortopædkirurgisk afd., Kolding Sygehus, Denmark; ${ }^{2}$ Sports Orthopaedic Research Centre-Copenhagen, Denmark; ${ }^{3}$ Ortopædkirurgisk afd., Vejle Hospital, Denmark

10.1136/bjsports-2019-scandinavianabs.33
Introduction To study if Diabetes Mellitus (DM) affects the severity of a frozen shoulder prognosis.

Methods In this 2 year follow-up study patients from three participating hospitals with newly diagnosed frozen shoulder were consecutively included and asked to fill out a questionnaire: at time of diagnosis and at 6-, 12- and 24 months follow-up. The questionnaire included the Oxford Shoulder Score (OSS) and the Visual Analogue Scale (VAS) for both maximum and average daily pain. Furthermore, patients were asked to report on whether arthroscopic release had been performed. DM status was recorded for all patients, and patients not previously diagnosed with DM were invited to be tested with the HbA1c test.

Results A total of 235 patients were included, of which 34 were previously diagnosed with frozen shoulder. Patients with DM had similar OSS $(p=0.22)$ and VAS score for maximum $(p=0.46)$ and average $(p=0.46)$ daily pain at time of diagnosis compared to patients without DM. Both groups improved in OSS and VAS score, but patients with DM had a worse OSS at 6 month $(\mathrm{p}=0.04)$, and 24 months follow-up $(\mathrm{p}=0.02)$; worse VAS score for maximum daily pain at 6 month $(p=0.04), 12$ month $(p=0.03)$ and 24 months follow-up $(p=0.03)$; and worse VAS score for average daily pain at 6 months $(p=0.02)$, and 12 months follow-up $(\mathrm{p}=0.01)$

Patients with DM had an increased probability of undergoing arthroscopic release $(\mathrm{p}<0.05)$.

Conclusion Patients with DM have a worse prognosis of their frozen shoulder, but all patients can expect gradually improvement during the 2 years following their diagnosis. 